\title{
Study on Innovative Measures to Strengthen Process Control of Outsourced Scientific Research Projects
}

\author{
Xinmei $\mathrm{Li}^{1, \mathrm{a},{ }^{*} \text {, Yufeng Chen }}{ }^{1, \mathrm{~b}}$, Huadong $\mathrm{Li}^{1, \mathrm{c}}$, Lei Zhang ${ }^{1, \mathrm{~d}}$, Suhong Chen ${ }^{1, \mathrm{e}}$, \\ Yong Zhang ${ }^{1, f}$, Xingang Zhou ${ }^{1, g}$
}

${ }^{1}$ State grid shandong electric power research institute, jinan, Shandong,China, 250002

alixinmei128@163.com, bchenyufeng169@sina.com, Ihdlhd@sohu.com, d18660130685@163.com, e285999524@qq.com, ${ }^{f}$ wanzhangyong@163.com, ${ }^{9} 13705319551 @ 163 . c o m$

\author{
*lixinmei128@163.com
}

Keywords: scientific research projects; outsourced research; process control; working mechanism.

\begin{abstract}
The rules and regulations established by the State Grid and its provincial branches have served as the framework for procedures regulating outsourcedscientific research projects, including project bidding, contract management, implementation and acceptance. Efforts have been made to explore the credit evaluation mechanism and the overall working mechanism involving various functional departments to standardize the outsourced project, further improving the efficiency and quality of R\&D.
\end{abstract}

\section{Introduction}

Restrictions factors such as resources and costs necessitate the outsourcing of certain scientific research projects to external companies that are rich in resources. By collaboration with these institutions and integration of optimal resources, the company manages to lower the costs, improve efficiency and adapt to the changing environment [1-3]. The cooperation mainly includes technology development, design, equipment trial-production, outsourcing testing, and leasing of instrument and equipment [4], which accounts for a relatively large proportion of the total funding of scientific research projects with a huge impact on the risk prevention and control of projects.

Recently, many management-related problems have been found in outsourced project. In some projects, most core technologies are outsourced instead of being developed or mastered by internal personnel, making it difficult to cultivate talents through project development. Meanwhile, a lack of supervision and regulation over the outsourcing institutions lead to lower efficiency of certain projects as reflected by compromised quality and poor application of the results, which is very detrimental to its promotion in the market. Therefore, it is necessary to further strengthen process control of outsourced scientific research projects along with enhanced internal control to standardize and improve the management of outsourced scientific research projects and prevent risks. Based on the characteristics of outsourced projects and the actual needs of the institution concerned, this paper proposes measures to enhance supervision over outsourcing institutions and a credit evaluation mechanism to improve the quality and efficiency of management and development of outsourced projects.

\section{Measures to enhance management of outsourced scientific projects}

\subsection{Formulation of project management plan for outsourced projects as a guidance for standard management}

In combination with the conditions of outsourced project as well as regulations of the State Grid and its provincial branches [5][6], a management plan for outsourced project has been compiled and issued by the Ministry of Science and Technology Committee, specifying procedures for management, bidding, acceptance and evaluation as well as responsibilities and obligations of personnel involved to establish a unified, standard and clear workflow as guidance and basis to fulfill expectations for outsourced projects. 


\subsection{Planning and bidding management of outsourced scientific projects}

Organize and submit plans for outsourced projects to determine the needs for outsourcing at the beginning of the year. Based on the bidding arrangement, technical specification of the outsourcing project shall be compiled by department in charge of the project. Efforts will be intensified to examine the core content of the project, the technical specifications while balancing the proportion of external institution-assisted tests against the number of outsourcing projects. The approved project plan shall be submitted to the Materials Department at provincial level, which will arrange bidding events.

\subsection{Regulation and control of outsourced scientific projects}

Regulation and control of outsourced scientific projects should be further standardized and improved, as shown in Figure 1.

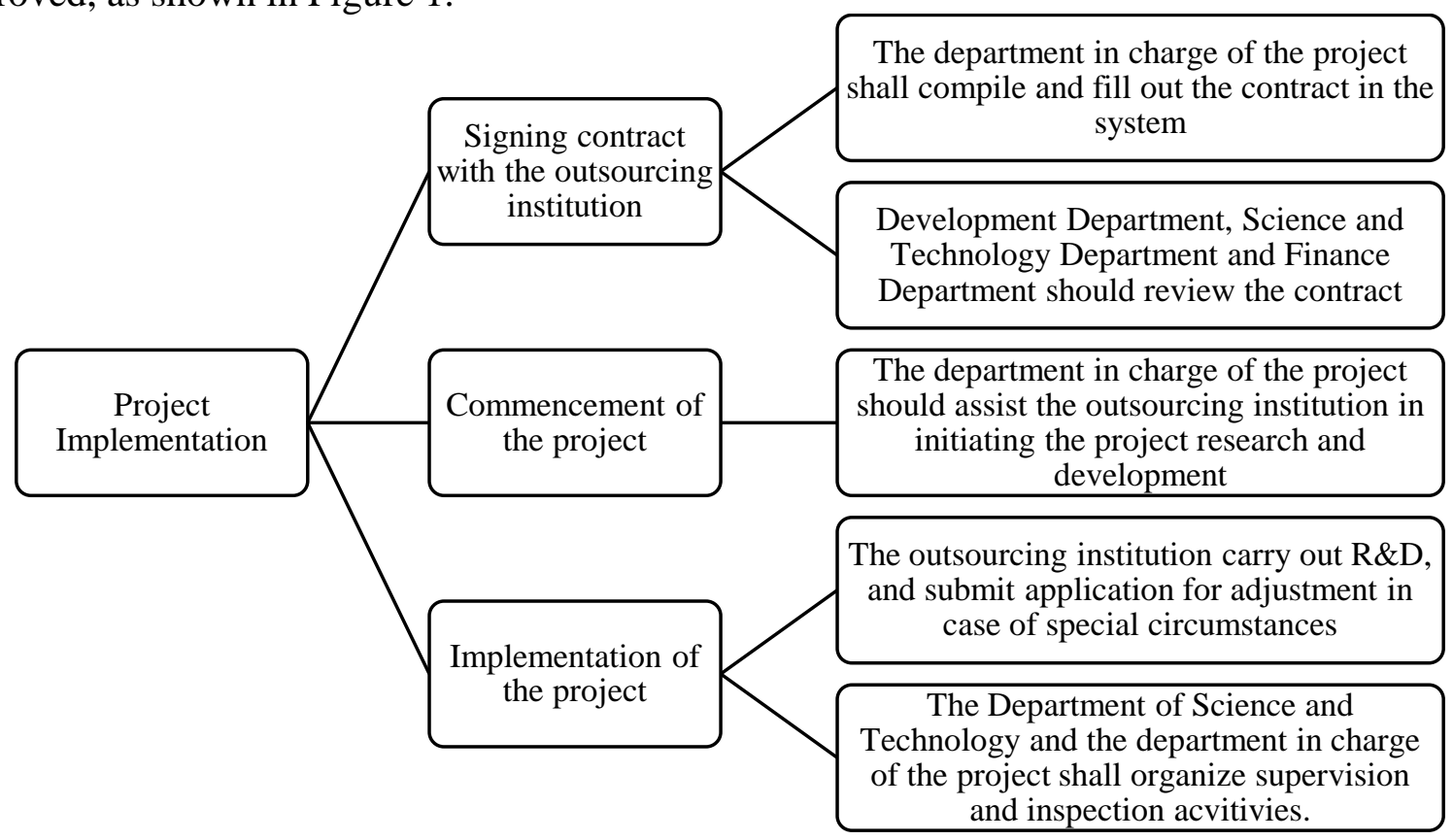

Figure 1 Planning and Management Procedures of Outsourced Projects

Based on the bidding results issued by the Materials Department at the provincial level, contracts for outsourcing the project shall be compiled and submitted in a timely manner for all departments concerned to review the terms in the written contract within 30 days after receiving the notification of winning the bid ${ }^{[7]}$. Remind and supervise projects that progress slowly, and evaluate projects that fail to complete the contract transfer and signing within the specified time.

The outsourcing institution shall organize implementation of the project based on the signed contract while the department in charge of the project shall supervise and inspect the progress of the project, in particular progress of top-priority projects which require inspection by the experts from the Department of Science and Technology. The progress of the project and any major event with possible impact on the completion of the project during the execution of the contract should be informed to the department in charge, which should submit the report to the Department of Science and Technology for approval for modification of the contract, adjustment of the plan or termination of the contract. The project shall not be altered without approval.

\subsection{Acceptance of outsourced projects}

The acceptance of outsourced projects is based on the management scheme, as shown in Figure 2. According to the requirements of the project acceptance criteria, a duplicate checking of the technical summary report is carried out, with the co-selection rate not exceeding $20 \%$. For those that fail the test, the project will not be accepted. For outsourced projects that pass the duplication checking, the project acceptance meeting will take place, inviting senior experts in the field to evaluate the project based on items and anticipated target. The outsourcing institution shall report 
the progress of the project for evaluation and inquiry from experts to determine whether it would be accepted, or if there is the need to correct existing problems within a time limit.

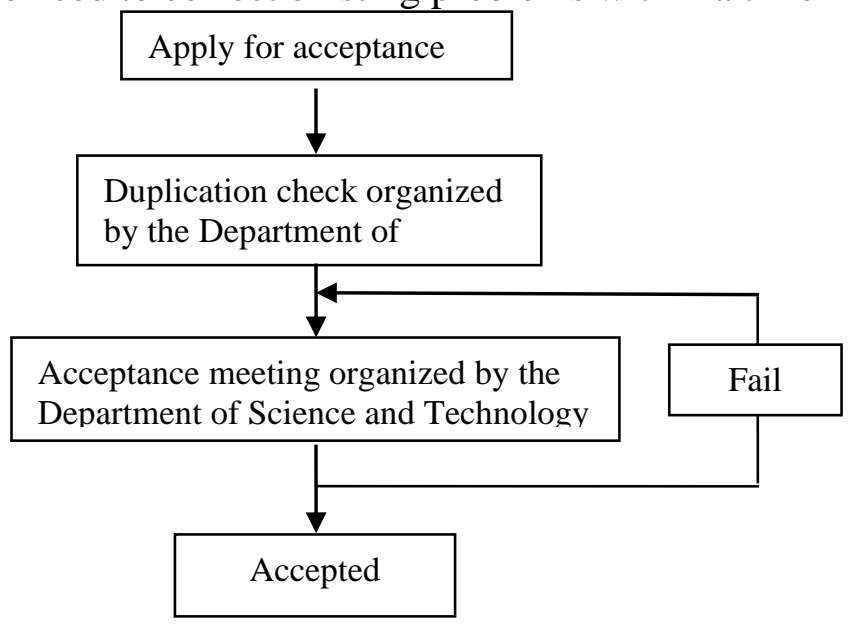

Figure 2 Acceptance Procedures of Outsourced Projects

\subsection{Credit management mechanism of outsourced projects by the Department of Science and Technology}

Based on the evaluation criteria of outsourced projects by the Department of Science and Technology along with standard of insincere behavior, a credit evaluation management mechanism is established for outsourced projects to evaluate tender, contract signing, project implementation, concluding inspection and acceptance of the whole process. The outsourcing institution could be warned, deprived of application qualification, terminated for project implementation or asked to return the funds as a punishment ${ }^{[6]}$. Meanwhile, the progress of the project will be incorporated into annual performance evaluation as an important reference.

\section{Achievements}

Through the above measures, the process management and control of outsourced scientific projects have been more standard and efficient, with remarkable progress mainly in the following aspects.

(1) The project management plan for outsourced scientific projects has been formulated as the guidance and basis. The issued guidelines for the acceptance of outsourced projects have clarified the standards and procedures for the acceptance of projects to ensure that there are rules to follow and rely on.

(2) The management procedures of outsourced projects have been established, including 1 first-level process, 4 second-level processes and 10 third-level processes. With the establishment of the framework, the responsibilities of functional departments and professional centers have been clarified.

(3) Standardized management further improves the planning of outsourced projects, with the submission, transfer and signing of contracts timelier and more efficient, and the control and supervision more specific, the concluding acceptance more accurate, allowing the department in charge of the project to better control the implementation of the project.

(4) Enhance risk control as it clarifies the responsibilities and obligations of each department in project bidding, contract signing, project implementation and acceptance of key process. The departments involved know their positions better as they spend more time studying the plan with an awareness of active defense and mutual supervision to improve their risk control capability, which provides a strong guarantee for project management of outsourced scientific projects.

\section{Conclusion}

According to the characteristics of outsourced projects in scientific research and development, innovative measures are proposed to strengthen the process control of outsourced projects in 
scientific research. It has formulated measures for the management of outsourced projects, the guidelines for its acceptance, and clarified the items, process and acceptance criteria. Measures have been taken to specify planning, bidding and purchasing, contract signing, project implementation and acceptance of each stage. By standardize the procedures, the risk of wasting fund has been reduced while the evaluation of outsourced projects is improved to further promote its application in the market.

\section{References}

[1] Y. L. Wang, P. F. Li, Analysis on the management of the outsourcing tasks of scientific research projects , Science, vol.1, pp.23-24, 2018.

[2] H. G. ZHAO, Contract risk prevention in the process of marketing scientific research outsourcing of Petroleum Enterprises, CHINA petrochem, vol.2, pp. 125-126, 2017.

[3] Y. H. Li, H. Q. Zheng, Study on management of research \&development funds in power Grid copmanies, Energy technology and economics, vol.1, pp.51-54, 2010.

[4] State Grid (science and technology / No.3), Implementation rules for budget preparation of science and technology projects of State Grid Corporation of China, 264-2017.

[5] State Grid (science and technology / No.3), Management rules for implementation and acceptance of science and technology projects managed by the headquarters of State Grid Corporation of China, 110-2018.

[6] State Grid (science and technology / No.2), Administrative measures for science and technology projects of State Grid Corporation of China, 263-2014.

[7] Order No. 86 of the president of the people's Republic of China, Law of the people's republic of China on tenders and bids, article 46, 2017. 